\title{
Genetic and epigenetic insight into morphospecies in a reef coral
}

James L. Dimond ${ }^{1,2, *}$, Sanoosh K. Gamblewood ${ }^{2}$, Steven B. Roberts ${ }^{1}$

${ }^{1}$ School of Aquatic and Fishery Sciences, University of Washington, 1122 NE Boat St., Seattle, WA 98105

USA

${ }^{2}$ Shannon Point Marine Center, Western Washington University, 1900 Shannon Point Rd., Anacortes, WA 98221 USA

Keywords: reef coral, RADseq, epigenetics, DNA methylation, phenotypic plasticity

*Correspondence: School of Aquatic and Fishery Sciences, University of Washington, 1122 NE Boat St., Seattle, WA 98105 USA, p: +1 206-819-1316, f: +1 360-293-1083, email: jdimond@gmail.com

Running title: Reef coral genetics and epigenetics 


\section{Abstract}

2 Incongruence between conventional and molecular systematics has left the delineation of many species

3 unresolved. Reef-building corals are no exception, with phenotypic plasticity among the most plausible

4 explanations for alternative morphospecies. As potential molecular signatures of phenotypic plasticity,

5 epigenetic processes may contribute to our understanding of morphospecies. We compared genetic and

6 epigenetic variation in Caribbean branching Porites spp., testing the hypothesis that epigenetics-

7 specifically, differential patterns of DNA methylation-play a role in alternative morphotypes of a group

8 whose taxonomic status has been questioned. We used reduced representation genome sequencing to

9 analyze over 1,000 single nucleotide polymorphisms and $\mathrm{CpG}$ sites in 27 Porites spp. exhibiting a range

10 of morphotypes from a variety of habitats in Belize. We found stronger evidence for genetic rather than

11 epigenetic structuring, identifying three well-defined genetic groups. One of these groups exhibited

12 significantly thicker branches, and branch thickness was a better predictor of genetic groups than depth,

13 habitat, or symbiont type. Epigenetic patterns were more subtle, with no clear groups. The more thickly

14 branched individuals in one of the genetic groups exhibited some epigenetic similarity, suggesting

15 potential covariation of genetics and epigenetics. This covariation was further supported by a positive

16 association between pairwise genetic and epigenetic distance. We speculate that epigenetic patterns

17 are a complex mosaic reflecting inheritance and diverse environmental histories. Given the role of

18 genetics in branching Porites spp. morphospecies we were able to detect with genome-wide

19 sequencing, use of such techniques throughout the geographic range may help settle their phylogeny.

\section{Introduction}


Inconsistencies between conventional and genetics-based taxonomy are common across taxa

24 (Patterson et al. 1993). Morphological characters have long been the basis of systematics, yet they can

25 be misleading in cases such as convergent evolution or extensive phenotypic plasticity (Potter et al.

26 1997; Fukami et al. 2004; Fritz et al. 2007). While molecular phylogenetics has illuminated species

27 relationships by identifying and resolving some of these issues, uncertainties often persist, and

28 disagreement among molecular studies is not uncommon. The inferences made by molecular studies are

29 influenced by numerous factors such as the number and type of markers and the models used to

30 analyze them (Brocchieri 2001; Yang \& Rannala 2012). Moreover, the field of epigenetics has uncovered

31 novel mechanisms of phenotypic variation and inheritance that has led to reappraisal of the traditional

32 theory of molecular evolution (Noble 2015; Skinner 2015).

Reef-building corals exhibit substantial inter- and intraspecific variation in growth forms that has

34 proved particularly problematic for the taxonomic delineation of species (Fukami et al. 2004; Forsman et

al. 2009; Flot et al. 2011). Conventional taxonomy based on morphological features has obscured coral

phylogenies and in some cases overestimated species diversity revealed by way of genetic analyses

37 (Fukami et al. 2004; Forsman et al. 2010; Prada et al. 2014). In other cases, supposed ecomorphs of the same species have turned out to be sibling species (Knowlton et al. 1992), and genetic data have also uncovered cryptic species among similar growth forms of what was thought to be a single species

40 (Keshavmurthy et al. 2013; Schmidt-Roach et al. 2013). The confusion surrounding coral taxonomy has

41 consequences for coral conservation, because a species cannot be conserved and managed if it cannot

42 be defined. For example, 25 coral species are currently listed under the U.S. Endangered Species Act

43 (NOAA 2015), and the ambiguous taxonomic status of some of these species has necessitated revision

44 using molecular data (Forsman et al. 2010). 
taxonomy (Forsman et al. 2010; Flot et al. 2011; Keshavmurthy et al. 2013; Prada et al. 2014). Indeed, a

recent reciprocal transplant study confirmed the role of phenotypic plasticity in alternative

morphotypes of Pocillopora spp. (Paz-García et al. 2015). Morphological plasticity is likely to be

particularly prevalent and adaptive in corals due to their sessility, longevity, modularity, and

51 indeterminate growth (Jackson \& Coates 1986; Sebens 1987; Todd 2008). In a review of 26 studies that

52 tested for morphological plasticity in approximately 20 different species of reef corals, $92 \%$ of studies

53 documented evidence for plasticity (Todd 2008).

The expanding field of epigenetics may hold promise for understanding phenotypic plasticity in

corals. Epigenetic processes are increasingly recognized as molecular signatures of phenotypic variation

(Duncan et al. 2014). DNA methylation, the most widely studied epigenetic mark, involves the addition

of a methyl group to a cytosine, most commonly in the context of a $\mathrm{CpG}$ dinucleotide pair. Along with

other epigenetic features such as histone modifications and small RNA molecules, methylation patterns

can influence gene expression, though the mechanisms appear to be diverse, complex, and context-

61 itself, which change relatively little during an individual's lifetime, genome DNA methylation patterns

64 alternative phenotypes in a range of taxa (Kucharski et al. 2008; Fonseca Lira-Medeiros et al. 2010;

65 Smith et al. 2015, 2016; Schield et al. 2016). Among different morphotypes of mangroves living in

67 levels of epigenetic variation. In the genomes of two alternative morphotypes of threespine

68 sticklebacks, (Smith et al. 2015) identified 77 differentially methylated regions whose functions were 
71 were genetic differences (Skinner et al. 2014). Thus, an increased understanding of epigenetics may

72 revise definitions of biological diversity and evolution (Skinner 2015), with implications for future

73 conservation efforts.

The taxonomic status of branching corals of the genus Porites in the tropical Western Atlantic

75 has been uncertain. There are currently three recognized species: P. porites (Pallas, 1766), P. furcata

76 (Lamarck, 1816), and P. divaricata (Lesueur, 1820). Branch diameter and corallite features have

77 traditionally been used to define these species, and while one study found morphological variation to be

78 nearly continuous (Brakel 1977), other studies have identified morphological breaks, with some

79 supporting traditional taxonomic delineations (Weil 1992; Budd et al. 1994; Jameson 1997; Jameson \&

80 Cairns 2012). Molecular studies have also failed to reach consensus (Weil 1992; Budd et al. 1994;

81 Forsman et al. 2009; Prada et al. 2014), but earlier studies used allozymes, which could be influenced by

82 epigenetic effects. The most recent and thorough population genetic analysis found no support for

83 upholding the three named species, finding no significant variation between supposed species across 11

84 genetic markers and multiple geographic sub-regions (Prada et al. 2014). The authors acknowledged

85 that it is possible that their study overlooked diversity somewhere in the genome, but identified

86 phenotypic plasticity as a plausible explanation for their results. To further explore the potential source

87 of phenotypic variation in branching Porites spp., we compared genetic and epigenetic diversity in these

88 corals using restriction site associated DNA (RAD) sequencing.

89

90 Methods

91

92 Specimen collection and DNA extraction

93 Corals were collected in May 2016 within approximately $4 \mathrm{~km}$ of the Smithsonian Institution's

94 Carrie Bow Cay Field Station in Belize ( $\left.16^{\circ} 48^{\prime} 9.39^{\prime \prime} \mathrm{N}, 88^{\circ} 4^{\prime} 54.99^{\prime \prime} \mathrm{W}\right)$. In the field, the three Caribbean 
95 branching Porites spp. are distinguished primarily by branch diameter and habitat type, so collections

96 targeted a broad range of branch diameters $(6-26 \mathrm{~mm})$, depths $(0.5-17 \mathrm{~m})$ and habitats (mangrove to

97 forereef) to sample as much variation as possible. A total of thirty specimens were collected, but we

98 obtained sufficient sequence data from only 27 of these (Table 1). Branch tips were cut with shears and

99 placed in a conical tube. Within one hour of collection, specimens were transferred to tubes containing

100 salt-saturated DMSO (SS-DMSO) for DNA preservation (Gaither et al. 2011). Coral tissue preserved in SS-

101 DMSO began to slough off the skeleton after several days and was easily removed using forceps. Small

102 pieces of tissue ( $0.5 \mu$ l volume) were washed three times via centrifugation with phosphate buffered

103 saline prior to DNA extraction using Qiagen DNeasy Blood and Tissue kits according to the

104 manufacturer's protocol, with an overnight lysis with proteinase K. Samples were further purified via

105 overnight ethanol precipitation, followed by resuspension in Qiagen AE buffer. Genomic DNA was

106 checked for yield and quality via fluorescence (Qubit BR assay) and gel electrophoresis, respectively.

107 The remaining coral skeletons were soaked in a $10 \%$ bleach solution for $24 \mathrm{hrs}$, then dried at 40

$108{ }^{\circ} \mathrm{C}$ for $48 \mathrm{hrs}$. The diameter of branch tips at their widest cross-section was measured to the nearest 0.5

109 mm with vernier calipers.

Symbiont genotyping

Reef corals commonly engage in species-specific associations with symbiotic dinoflagellates

113 (Symbiodinium spp.), and these associations can also be related to genetic structure within a given host

114 (Bongaerts et al. 2010; Finney et al. 2010). To identify the dominant Symbiodinium type associated with

115 each coral, an approximately 700 base pair region of domain $\mathrm{V}$ of the cp23S-rDNA region was PCR-

116 amplified using primer pair 23S1 (5'-CACGACGTTGTAAAACGACGGC TGTAACTATAACGGTCC-3') and 23S2

117 (5'-GGATAACAATTTCACACAGGCCATCGTATTGAACCCAGC-3') (Santos et al. 2002). PCR was performed in

$11825 \mu$ l volumes containing $1 \mathrm{X}$ green buffer (Promega), $2.5 \mathrm{mM} \mathrm{MgCl}_{2}, 240 \mu \mathrm{M}$ dNTP, $5 \mathrm{pmol}$ of each 
primer, 1 U Taq (GoTaq, Promega), and 1-20 ng of template DNA. Reactions were carried out in an

120 Applied Biosystems Veriti thermocycler under the following conditions: initial denaturing period of 1 min

121 at $95{ }^{\circ} \mathrm{C}, 35$ cycles of $95^{\circ} \mathrm{C}$ for $45 \mathrm{~s}, 55^{\circ} \mathrm{C}$ for $45 \mathrm{~s}$, and $72{ }^{\circ} \mathrm{C}$ for $1 \mathrm{~min}$, and a final extension period of 7

122 min. PCR products were cleaned (NEB Monarch kit) and checked on a 1\% agarose gel. Cleaned PCR

123 products $(10 \mathrm{ng} / \mu \mathrm{L})$ were sent to Sequetech Corporation (Mountain View, $\mathrm{CA}$ ) for Sanger sequencing

124 using the forward primer. Chromatograms were edited with Geneious v9.1.5, converting bases with a

125 Phred score $<30$ to Ns. Sequences were then aligned using ClustalW and queried against the GenBank

126 nucleotide database in Geneious.

RAD library preparation and sequencing

Double digest RADseq (ddRADseq) libraries were prepared following the methods of (Peterson

130 et al. 2012). Only samples with high molecular weight DNA were used for library preparation. For each

131 sample, a minor variation of ddRADseq called EpiRADseq was also employed to evaluate methylated loci

132 (Schield et al. 2016). Both methods use two restriction enzymes-a rare cutter and a common cutter-

133 to perform a double digest of the DNA at specific restriction sites throughout the genome, with the only

134 difference that EpiRADseq uses a methylation-sensitive common cutter that will not cut methylated loci.

135 Both methods used the rare cutter PstI (5'-CTGCAG-3' recognition site), while the common cutter MspI

136 (5'-CCGG-3' recognition site) was used for ddRADseq and the methylation-sensitive isoschizomer Hpall

137 (also 5'-CCGG-3' recognition site) was used for EpiRADseq preparations. Double digests of 300-500 ng

138 gDNA per sample were carried out using 20 units of each enzyme in the manufacturer's supplied buffer

139 (New England Biolabs, NEB) for 5 hours at $37^{\circ} \mathrm{C}$. Samples were cleaned using magnetic beads (Sera-Mag

140 SpeedBeads) prior to ligation of barcoded Illumina adapters onto the fragments (Peterson et al. 2012).

141 After two rounds of bead cleanup, samples were pooled into 12 libraries (along with 18 other samples

142 not analyzed in this study), which were then subjected to automated size-selection of fragments 
143 between 415 and 515 bp using a Pippin Prep (Sage Science). Libraries were then PCR amplified using

144 Phusion Taq (NEB) and Illumina-indexed primers (Peterson et al. 2012). Final library fragment sizes and 145 concentrations were evaluated with D1000 ScreenTape on an Agilent 2200 TapeStation. Libraries were 146 sent to the Vincent J. Coates Genomics Sequencing Laboratory at the University of California, Berkeley, 147 where their concentrations were verified via qPCR prior to $100 \mathrm{bp}$, paired-end sequencing in equimolar 148 ratios on the Illumina HiSeq 4000.

RAD sequence assembly

Sequences were assembled using ipyrad v0.3.41 (Eaton 2014). We used the 'denovo - reference'

152 assembly method with the Symbiodinium minutum (clade B; GenBank accession GCA_000507305.1

153 (Shoguchi et al. 2013)) and Symbiodinium kawagutii (clade F;

154 http://web.malab.cn/symka_new/data/Symbiodinium_kawagutii.assembly.935Mb.fa.gzb.fa.gz (Lin et al.

155 2015) ) genomes used as reference to subtract symbiont reads from the de novo assembly. We

156 concatenated these genomes into a single reference file. Step one of the ipyrad workflow demultiplexed

157 the data in each pool by identifying restriction overhangs and barcode sequences associated with each

158 sample; zero barcode mismatches were tolerated. Demultiplexed samples were then combined in a

159 single directory for further steps. In step two, reads were trimmed of barcodes and adapters and quality

160 filtered using a q-score threshold of 20 , with bases below this score converted to Ns and any reads with

161 more than $5 \mathrm{Ns}$ removed. Step three mapped reads to the concatenated symbiont reference genomes

162 with BWA using the default bwa mem setting and removed any mapped reads. With the remaining

163 reads, similar clusters of reads were identified using a threshold of $85 \%$ similarity and aligning them. We

164 chose $85 \%$ as a moderately conservative clustering threshold to avoid over-splitting of loci (Harvey et al.

165 2015). Next, step four performed joint estimation of heterozygosity and error rate (Lynch 2008) based

166 on a diploid model assuming a maximum of 2 consensus alleles per individual. Step five used the 
parameters from step four to determine consensus bases calls for each allele, and removed consensus

sequences with greater than 5 Ns per end of paired-end reads. With consensus sequences identified,

171 SNPs per locus (20), maximum proportion of shared heterozygous sites per locus (0.5), and minimum

172 number of samples per locus (15).

Henceforth, we will use the term locus to refer to a consensus paired-end read. The term SNP

174 refers specifically to a single nucleotide polymorphism on a locus, while the term $C p G$ refers to a

175 cytosine-guanine dinucleotide pair that can be either methylated or non-methylated at the 5'-CCGG-3'

176 restriction site of each locus.

\section{SNP analysis}

We analyzed unlinked SNPs that were sampled by ipyrad at 1 SNP per locus with the least

amount of missing data; SNPs were sampled randomly if they had equal amounts of missing data. We

181 were able to estimate the SNP error rate, defined as the proportion of SNP mismatches between pairs of

182 the same individuals (Mastretta-Yanes et al. 2015), by treating ddRADseq and EpiRADseq samples as

183 technical replicates and computing pairwise differences between individuals using the dist.gene function

184 in the R package ape (Paradis et al. 2004). We then analyzed a SNP dataset from ddRADseq libraries that

185 had no missing data across samples. While this reduced the dataset considerably, it allowed us to test

186 variation across a similar set of loci for both the genetic and epigenetic analysis, as explained further

187 below.

188 Genetic differentiation among samples was examined using multidimensional scaling $(\mathrm{k}=2)$

189 using the $c m d s c a l e ~ R$ function. Discriminant analysis of principal components (DAPC) in the R package

190 adegenet (Jombart 2008) was used to further examine these patterns. Rather than applying prior 
assumptions about the identity of each coral specimen to assign population groups, we used the

find.clusters function in adegenet to identify groups. This k-means clustering function reduces the data with principal components analysis (PCA) before estimating the number of clusters with the lowest Bayesian information criterion (BIC). All PCs were retained for the analysis and a maximum of 10 clusters was specified. Once groups were identified, cross-validation (xvalDapc) was used to estimate the

196 number of PCs to retain for the subsequent DAPC analysis. In the groups identified by the DAPC analysis, 197 genetic differentiation between groups was evaluated by computing pairwise Weir and Cockerham's $F_{S T}$ using the R package hierfstat (Goudet 2005).

DNA methylation analysis

Methylation detection with EpiRADseq data involves analysis of read counts (Schield et al. 2016). Presence of a given read in the EpiRADseq dataset indicates that the locus is not methylated at the restriction cut site. Conversely, if a locus is methylated at the 5'-CCGG-3' cut site, Hpall is blocked

204 from cutting, and the locus will be absent from the dataset. Hence, read counts of zero are informative,

205 but they could also be the result of missing data through processes such as allele dropout or variation in 206 library size or fragment size selection. To control for this, we used ddRADseq data to normalize the 207 EpiRADseq data. Any loci with zeros in the ddRADseq library were treated as absent and removed, 208 thereby leaving zeros in the EpiRADseq library only where the locus was counted in the ddRADseq 209 library. This had the added benefit that it resulted in analysis of a similar set of loci as the SNP analysis 210 described above. EpiRADseq and ddRADseq read counts per locus were highly correlated, with the exception of 212 methylated loci, which, as expected, had low or zero abundances in EpiRADseq libraries (Fig. 1A). The 213 dataset comprising read counts for both EpiRADseq and ddRADseq was subject to normalization of read 214 counts using TMM normalization in the R package edgeR (Robinson et al. 2010). Residuals of linear 
215 regressions of EpiRADseq and ddRADseq libraries provided optimal differentiation of methylated loci

216 from non-methylated loci (Fig. 1B\&C). Finally, a binary dataset was created from these data by setting a

217 residual threshold of -1 for methylated loci such that $1=$ methylated and $0=$ non-methylated (Fig. 1C).

218 These data were evaluated using multidimensional scaling as described above for the SNP analysis.

219 EpiRADseq read counts could theoretically represent variable levels of methylation within a

220 sample, such as through variation in methylation across pooled replicates, or among different tissue or

221 cell types (Schield et al. 2016). However, our analysis of ddRADseq and EpiRADseq read counts in

222 tandem indicated that much of the variability in read counts occurs in both libraries and is thus likely

223 related to library preparation effects such as fragment size-selection or PCR bias (Davey et al. 2013). In

224 the original EpiRADseq method developed by Schield et al. (2016), PCR effects were minimized by using

225 unique molecular identifier sequences attached to fragments. We suspect that fragment size-selection

226 effects could be equally important in driving read count variability. For example, abundant reads could

227 simply represent the mean or mode fragment size within the library after size-selection, while less

228 abundant reads could represent the tails of the distribution. In either case, our analysis suggests that

229 much of the read count variation above zero does not reflect methylation levels, and by normalizing

230 EpiRADseq data to the ddRADseq data and creating a binary dataset, we removed much of the potential

231 bias described above.

232 A repository with the complete bioinformatic workflow described above can be accessed at

233 https://github.com/jldimond/Branching-Porites.

\section{Results}


An average of 3.24 million paired-end reads per sample were obtained based on restriction

239 overhangs and barcodes, with an average of 2.63 million reads per sample remaining after q-score and

240 adapter filtering. After filtering for symbionts and minimum read depth, an average of 26,330 consensus

241 loci per sample were obtained, with a total of 135,980 unique consensus loci across samples. Obtaining

242 datasets with no missing data across samples substantially reduced the number of loci; the final SNP

243 dataset consisted of 1,113 unlinked SNPs (1,113 consensus paired-end loci with 1 SNP sampled per

244 locus) across 27 samples, while the methylation dataset consisted of $1,712 \mathrm{CpGs}(1,712$ consensus

245 paired-end loci with one CpG cut site per locus) across 25 samples. Differences in the number of

246 SNPs/CpGs between datasets were due to the different numbers of samples; two samples, 101 and 112,

247 were removed from the methylation dataset due to low coverage. This reduced the number of samples

248 in the methylation dataset but resulted in a greater number of shared $\mathrm{CpG}$ sites.

Symbiont identity

BLAST searches of Symbiodinium cp23S sequences indicated that the dominant symbiont in the

252 majority of corals was a clade C Symbiodinium with high similarity to Symbiodinium subclade C3 (Table

253 1). Four corals occurring in shallow water habitats ([ $2.7 \mathrm{~m}$ ) hosted clade A Symbiodinium with high

254 similarity to subclade A3. We were unable to amplify cp23S in three of the specimens despite repeated

255 attempts.

257 Genetic patterns

Based on SNP mismatches between technical replicates, the SNP error rate was estimated to be

$2593.6 \%$ (standard deviation, SD, 3.1\%), which is on the lower end of the range that has been reported

260 previously (Mastretta-Yanes et al. 2015) (Fig. 2). In other words, multilocus genotypes of technical

261 replicates were $96.4 \%$ similar on average. In contrast, nearly all non-replicate pairwise comparisons 

exhibited similarity within the range of the SNP error (Fig. 2). The high similarity of these individuals suggests they are possible clones. Specimens 109 and 114 were separated by approximately $45 \mathrm{~m}$, while specimens 127 and 115 were separated by approximately $450 \mathrm{~m}$. If they are clones, these distances make it unlikely that they resulted from fragmentation. with samples clustering into three generally well-separated groups (Fig. 3). K-means clustering of SNPs showed the strongest support for three clusters according to BIC values (Fig. 4A). Based on results of cross-validation, nine PCs (the maximum suggested given the sample size) were retained for DAPC

271 analysis of the three groups, which resulted in two discriminant axes that were both retained (Fig. 4B

272 inset). As with the MDS analysis, DAPC indicated clear separation of the three SNP groups (Fig. 4B). The 273 two pairs of potential clones were in two separate groups (109 \& 114 in group 2, 127 \& 115 in group 1).

274 Similar values of $F_{S T}$ were observed between groups ( $F_{S T}$ for each pairwise comparison: $1 \& 2=0.194 ; 1 \& 3$ $275=0.207 ; 2 \& 3=0.191)$. We evaluated potential factors associated with genetic structure using multiple regression. For 277 the genetic variable, we used the first discriminant axis from the DAPC analysis of SNPs. This was 278 regressed against collection depth, symbiont type, habitat, and branch diameter. The model explained $27961 \%$ of the variation in the SNP variable. The function calc.relimp in the R package relaimpo was used to 280 estimate the relative importance of each model component. The relative importance of depth, symbiont 281 type, habitat, and branch diameter in the model was $0.5 \%, 2 \%, 31 \%$, and $66.5 \%$, respectively. We further 282 evaluated branch diameter with a one-way ANOVA, followed by pairwise t-tests with Bonferroni 283 adjustment. Group 1 branch diameter was significantly different from both groups 2 and $3(p<0.001)$, 284 while groups 2 and 3 were not significantly different from each other ( $p=0.724)$ (Fig. 5). 
Among the $1,712 \mathrm{CpGs}$, a range of 314-360 were methylated per sample, yielding a mean methylation level of $19.5 \%$ (SD $0.008 \%$ ). However, there were two outliers with high levels of methylation. This was due largely to low read count loci being categorized as methylated. A more

290 stringent minimum read count threshold of 10 reads per locus was therefore applied to the ddRADseq 291 dataset and this resulted in 1,368 CpGs with a range of 238-263 methylated CpGs per sample, corresponding to a methylation level of $18.3 \%$ (SD 0.005\%). Of the 1,368 CpGs, 208 were differentially methylated, 131 were constitutively methylated across all samples, and 1029 were constitutively non-

294 methylated across all samples.

300 groups identified in the SNP analysis, individuals in group 1 appeared to cluster adjacent to each other

301 (far left color swatch, Fig. 7). These also tended to be samples with thicker branch diameter (bar plot,

302 Fig. 7).

Linkages between genetic and epigenetic variation

SNPs with high contributions to the DAPC analysis were linked to differentially methylated CpGs (i.e.,

307 from the same RAD loci). Unique locus IDs generated by ipyrad were used to merge differentially 
difference between contribution scores associated with differentially methylated loci and scores from a random sample of the data (Kolmogorov-Smirnov test; $p=0.365 ;$ Fig. 8).

312 epigenetic distances, again using the dist.gene function in the R package ape. This was the same statistic

313 generated in Fig. 2 for the SNP data, measuring the proportion of pairwise mismatches in multilocus

314 SNPs and CpG methylation status. The majority of pairwise comparisons did not exhibit a clear

315 association between genetic and epigenetic distance (Fig. 9). However, the two pairs of genetic outliers

316 identified earlier with very low genetic distances ( $109 \& 114,127 \& 115)$ also had the lowest epigenetic

317 distances. A linear regression of these data was significant $(p<0.001)$.

Discussion

Our sample of branching Porites spp. from Belize exhibited clear genetic differentiation,

322 supporting the idea that these corals comprise three separate and fairly well-defined groups. While this

323 result is in general agreement with older studies based on allozymes (Weil 1992; Budd et al. 1994), it

324 contrasts with the most recent study based on 11 genetic markers (Prada et al. 2014). The inferences we

325 were able to make likely reflect the large number of markers we sampled. Reduced representation

326 genome sampling methods like RADseq have shown great promise in resolving phylogenetic

327 relationships, especially in recalcitrant taxa like cnidarians for which traditional mitochondrial and

328 nuclear markers have shown limited success (Pante et al. 2015; Combosch \& Vollmer 2015; Herrera \&

329 Shank 2016; Rosser et al. 2017). Despite the number of markers we sampled, however, our study lacks

330 sufficient geographic breadth to draw strong phylogenetic conclusions. Indeed, all evaluations of

331 branching Porites spp. to date, including ours, have been limited by geographic scope, insufficient colony

332 sample size, or low genome coverage. A thorough reexamination of branching Porites spp. phylogeny 
across the Caribbean / Western Atlantic region may be warranted, assessing both genome-wide

334 variation and multivariate morphological traits. Ideally, such a study would incorporate randomized collections in different habitats to permit assessment of potential habitat selection.

337 that the genetic differentiation we observed is associated with colony-level morphological variation.

338 Variation in branch thickness is the primary diagnostic feature used for Porites spp. identification in the field, and is likely what historically prompted delimitation of species groups. Corallite-level variation in morphology is also well documented, and most studies using these methods have identified distinct 341 groups based on multivariate corallite characters (Weil 1992; Budd et al. 1994; Jameson 1997). Two of these studies found evidence supporting the three recognized species (Weil 1992; Jameson 1997), while one found up to five morphospecies among specimens collected from three regions (Budd et al. 1994),

344 and another identified continuous variation without any clear breaks (Brakel 1977). However, despite

345 the continuous variation observed by Brakel (1977), he judged the variation to be largely genetic (fixed)

346 based on the observation that 1) corals from very different environments can have very similar

347 morphologies, and 2) corals from very similar environments can have very different morphologies. This 348 concurs with our analysis suggesting that morphotype variation in Porites spp. has a genetic basis. On

349 the other hand, we also observed evidence for phenotypic plasticity, such as in the case of three 350 specimens collected from nearby mangrove prop roots exhibiting similar branch morphologies; one of 351 these colonies (122) was assigned to group 2 while the others $(118,121)$ were assigned to group 3. Based on the lack of consensus among previous studies concerning the taxonomic status of 353 branching Porites spp., and hypotheses that phenotypic plasticity might underlie this incongruence, we 354 did not anticipate finding strong genetic differentiation and instead we hypothesized that epigenetic 355 patterns might prove more informative. Instead, patterns of DNA methylation were less conclusive than 356 genetic patterns. Levels of methylation did not vary greatly among samples, and even among 
differentially methylated CpGs, most were either methylated or unmethylated in a majority of samples.

Similarly, little variation in methylation was observed in a recent study of threespine sticklebacks, with

only 737 differentially methylated CpG sites identified out of $1,445,567$ sites examined across eight

individuals (Smith et al. 2015). However, these differentially methylated loci were associated with

alternative phenotypes (Smith et al. 2015). The only phenotypic pattern to emerge from our data was a suggestion that individuals in group 1 , which also tended to be more thickly branched, were generally grouped close together. Thus, there is an intriguing possibility that epigenetic variation is coupled with genetic variation in these individuals. genetic and epigenetic distance. This relationship implies a potential heritable component to the methylation patterns, although it was strongest when including two pairs of outliers. The physical distances separating these pairs suggests that they are unlikely to be clones resulting from fragmentation, but possibly clones from asexually produced larvae (Harrison 2011). The interpretation that these pairs are clones is more likely, because even though some branching Porites spp. colonies are

371 hermaphrodites capable of self-fertilization (Schlöder \& Guzman 2008), Mendelian segregation leads to an extremely low probability of identical parental and offspring genotypes (Stoddart 1983). If they are

373 indeed clones, the similar methylation patterns in these individuals would reflect inheritance via mitosis

374 rather than sexual recombination through the germline. However, regardless of whether methylation

375 profiles were transmitted sexually or asexually in the two pairs of outliers, it is notable that while

376 genetic distance was near zero, epigenetic distance was relatively high. This could reflect divergence of

377 the methylome due to environmental effects. Methylation levels in corals have recently been shown to

378 be at least partially under environmental influence over short time scales (Putnam et al. 2016). Clearly,

379 the methylation patterns we observed in Porites spp. exhibit a high degree of noise, and this noise may

380 reflect the diverse habitat conditions and environmental histories experienced by the corals analyzed 
here. Environmental influence is also suggested by the case of the three corals collected from adjacent mangrove prop roots $(118,121,122)$; while these corals came from two different genetic groups, they were clustered closely to each other epigenetically.

Beyond simply covarying with genetic variation, some studies have reported epigenetic variation potentially driving genetic variation (Skinner et al. 2015; Smith et al. 2016). In our analysis, differentially methylated CpGs were not more likely to be linked to SNPs with strong contributions to genetic could be separated by up to 500 bp on the RAD loci we generated. Moreover, given the read lengths and profiling techniques, the number of $\mathrm{CPG}$ sites we were able to sample per locus with EpiRADseq was a fraction of the number of nucleotides we were able to assess for SNPs. For example, for a given pairedend locus, nearly 200 nucleotides were assayed for potential SNPs whereas only a single CpG site was assayed for methylation. Nonetheless, if we consider the EpiRADseq method a random sample of CpGs, the $18 \%$ methylation we observed is within the range expected for invertebrates (Zemach et al. 2010; Sarda et al. 2012; Dimond \& Roberts 2016). enhancing our ability to probe molecular processes, but they are not without error. Our analysis of 397 ddRADseq and EpiRADseq libraries in tandem provided robust control for error in both the SNP and 398 methylation datasets. Technical replicates have been advocated as a means to assess genotyping error, 399 and by assessing genotypes of the same individuals from the two libraries we confirmed that this error 400 was within the range documented elsewhere (Mastretta-Yanes et al. 2015; Recknagel et al. 2015). For 401 the methylation analysis, comparing EpiRADseq libaries to ddRADseq libraries was a key factor in 402 controlling for library composition effects, reducing the likelihood of false positives. This paired library 403 approach appears to be a strong alternative to the unique molecular identifier approach used by Schield 404 et al. (2016). 
In addition to genetic data, prevailing symbiont populations could provide an additional means

406 to evaluate whether branching Porites spp. exhibit species-level differentiation. Species-specific

407 associations between host corals and Symbiodinium are common in the Caribbean, particularly among

408 brooding species such as Porites (Finney et al. 2010; Bongaerts et al. 2015). Furthermore, branching

409 Porites spp. appear to associate with host-specialist symbionts that are not common in other hosts

410 (Finney et al. 2010; Bongaerts et al. 2015). Branching Porites spp. also tend to host distinct symbionts

411 from their congener P. astreoides (Finney et al. 2010; Bongaerts et al. 2015). Although cp23S is not a

412 fine-scale genetic marker, the majority of corals we analyzed hosted the same Symbiodinium clade C

413 phylotype, while three individuals in group 3 and one in group 2 hosted the clade A phylotype (Table 1).

414 While host-symbiont pairings can be regionally specific (LaJeunesse 2002; Finney et al. 2010), if

415 branching Porites spp. have the same symbiont profile on a given reef regardless of potential differences

416 in host genetics or morphology, this could be an argument against considering them separate species. A

417 comprehensive reexamination of branching Porites spp. would be wise to include an assessment of

418 Symbiodinium communities.

Conclusion

Contrary to our expectations, branching Porites spp. morphotype variation was better explained

422 by genetic patterns than epigenetic patterns. This analysis benefited from the resolution afforded by

423 genome-wide sequencing, and may justify a more thorough analysis of branching Porites spp. phylogeny

424 throughout the tropical Western Atlantic. Although patterns of DNA methylation were not as conclusive

425 as genetic patterns, there was some evidence of covariation between genetic and epigenetic variation.

426 This possibility, as well as potential environmental influence on methylation in corals, will require

427 further study. Given the increasingly powerful molecular biology tools available for work in 
environmental epigenomics, stronger inferences about the extent, variability, and potential functions of

429 epigenetic processes in corals are only a matter of time.

\section{Acknowledgements}

We thank the Smithsonian Institution's Caribbean Coral Reef Ecosystems Program for field

434 support, and the Belize Fisheries Department for specimen export permitting. RADseq training and

435 materials were generously provided by Adam Leaché and Kevin Epperly. Daniel Thornhill and Terra

436 Hiebert provided advice on symbiont genotyping. Sam White, Hollie Putnam, Katherine Silliman, and

437 Megan Hintz provided helpful comments that improved the manuscript. This study was supported by

438 the Hall Conservation Genetics Research Award (UW-CoEnv), the ARCS Foundation Seattle Chapter, the

439 John E. Halver Fellowship (UW-SAFS), and National Science Foundation Award OCE-1559940.

\section{$441 \quad$ Data Accessibility}

443 -DNA sequences: GenBank accession numbers KY649212-KY649238; SRA accession numbers

444 SAMN06566335-SAMN06566364.

445 -Repository detailing analysis methods: https://github.com/jldimond/Branching-Porites

\section{Author Contributions}

J.D. conceived and designed the study, collected the specimens, prepared RAD libraries,

450 analyzed the data, and drafted the manuscript. S.G. processed the specimens, extracted DNA, and

451 performed the symbiont genotyping. S.R. contributed reagents, equipment and analysis tools. 


\section{Literature Cited}

Bongaerts P, Carmichael M, Hay KB et al. (2015) Prevalent endosymbiont zonation shapes the depth distributions of scleractinian coral species. Royal Society open science, 2, 140297.

Bongaerts P, Riginos C, Ridgway T et al. (2010) Genetic divergence across habitats in the widespread coral Seriatopora hystrix and its associated Symbiodinium. Plos One, 5, e10871.

Brakel W (1977) Corallite variation in Porites and the species problem in corals. Proceedings of the Third Internatioeedinal Coral Reef Symposium, 1, 457-462.

Brocchieri L (2001) Phylogenetic inferences from molecular sequences: review and critique. Theoretical Population Biology, 59, 27-40.

Budd AF, Johnson KG, Potts DC (1994) Recognizing morphospecies in colonial reef corals: I. Landmarkbased methods. Paleobiology, 20, 484-505.

Combosch DJ, Vollmer SV (2015) Trans-Pacific RAD-Seq population genomics confirms introgressive hybridization in Eastern Pacific Pocillopora corals. Molecular Phylogenetics and Evolution, 88, 154162.

Davey JW, Cezard T, Fuentes-Utrilla P et al. (2013) Special features of RAD Sequencing data: implications for genotyping. Molecular Ecology, 22, 3151-3164.

Dimond JL, Roberts SB (2016) Germline DNA methylation in reef corals: patterns and potential roles in response to environmental change. Molecular Ecology, 25, 1895-1904.

Duncan EJ, Gluckman PD, Dearden PK (2014) Epigenetics, plasticity, and evolution: How do we link epigenetic change to phenotype? Journal of Experimental Zoology. Part B, Molecular and Developmental Evolution, 322, 208-220.

Eaton DAR (2014) PyRAD: assembly of de novo RADseq loci for phylogenetic analyses. Bioinformatics, 30, 1844-1849.

Finney JC, Pettay DT, Sampayo EM et al. (2010) The relative significance of host-habitat, depth, and geography on the ecology, endemism, and speciation of coral endosymbionts in the genus Symbiodinium. Microbial Ecology, 60, 250-263.

Flot J, Blanchot J, Charpy L et al. (2011) Incongruence between morphotypes and genetically delimited species in the coral genus Stylophora: phenotypic plasticity, morphological convergence, morphological stasis or interspecific hybridization? BMC Ecology, 11, 22.

Fonseca Lira-Medeiros C, Parisod C, Fernandes R et al. (2010) Epigenetic variation in mangrove plants occurring in contrasting natural environment. Plos One, 5, e10326.

Forsman ZH, Barshis DJ, Hunter CL, Toonen RJ (2009) Shape-shifting corals: molecular markers show morphology is evolutionarily plastic in Porites. BMC Evolutionary Biology, 9, 45.

Forsman ZH, Concepcion GT, Haverkort RD et al. (2010) Ecomorph or endangered coral? DNA and microstructure reveal hawaiian species complexes: Montipora dilatata/flabellata/turgescens \& $\mathrm{M}$. patula/verrilli. Plos One, 5, e15021.

Fritz U, Hundsdörfer A, Široký P et al. (2007) Phenotypic plasticity leads to incongruence between morphology-based taxonomy and genetic differentiation in western Palaearctic tortoises (Testudo graeca complex; Testudines, Testudinidae). Amphibia-Reptilia, 28, 97-121.

Fukami H, Budd AF, Paulay G et al. (2004) Conventional taxonomy obscures deep divergence between Pacific and Atlantic corals. Nature, 427, 832-835.

Gaither M, Szabó Z, Crepeau M, Bird C, et al. (2011) Preservation of corals in salt-saturated DMSO buffer is superior to ethanol for PCR experiments. Coral Reefs, 30, 329-333.

Gavery MR, Roberts SB (2014) A context dependent role for DNA methylation in bivalves. Briefings in functional genomics, 13, 217-222.

Goudet J (2005) Hierfstat, a package for R to compute and test hierarchical F-statistics. Molecular ecology resources, 5, 184-186. 
Harrison PL (2011) Sexual Reproduction of Scleractinian Corals. In: Coral Reefs: An Ecosystem in Transition (eds Dubinsky Z, Stambler N), pp. 59-85. Springer Netherlands, Dordrecht.

Harvey MG, Judy CD, Seeholzer GF et al. (2015) Similarity thresholds used in DNA sequence assembly from short reads can reduce the comparability of population histories across species. PeerJ, $\mathbf{3}$, e895.

Herrera S, Shank TM (2016) RAD sequencing enables unprecedented phylogenetic resolution and objective species delimitation in recalcitrant divergent taxa. Molecular Phylogenetics and Evolution, 100, 70-79.

Jackson JBC, Coates AG (1986) Life cycles and evolution of clonal (modular) animals. Philosophical Transactions of the Royal Society B: Biological Sciences, 313, 7-22.

Jameson S (1997) Morphometric analysis of the Poritidae (Anthozoa: Scleractinia) off Belize. In: Proceedings of the 8th International Coral Reef Symposium (eds Lessios H, McIntyre I), pp. 15911596. International Society for Reef Studies, Smithsonian Tropical Research Institute, Panama.

Jameson S, Cairns S (2012) Neotypes for Porites porites (Pallas, 1766) and Porites divaricata Le Sueur, 1820 and remarks on other western Atlantic species of Porites (Anthozoa: Scleractinia). Proceedings of the Biological Society of Washington, 125, 189-207.

Jombart T (2008) adegenet: a R package for the multivariate analysis of genetic markers. Bioinformatics, 24, 1403-1405.

Keshavmurthy S, Yang S-Y, Alamaru A et al. (2013) DNA barcoding reveals the coral "laboratory-rat", Stylophora pistillata encompasses multiple identities. Scientific reports, 3, 1520.

Knowlton N, Weil E, Weigt LA, Guzmán HM (1992) Sibling Species in Montastraea annularis, Coral Bleaching, and the Coral Climate Record. Science, 255, 330-333.

Kucharski R, Maleszka J, Foret S, Maleszka R (2008) Nutritional control of reproductive status in honeybees via DNA methylation. Science, 319, 1827-1830.

LaJeunesse T (2002) Diversity and community structure of symbiotic dinoflagellates from Caribbean coral reefs. Marine biology, 141, 387-400.

Lin S, Cheng S, Song B et al. (2015) The Symbiodinium kawagutii genome illuminates dinoflagellate gene expression and coral symbiosis. Science, 350, 691-694.

Lynch M (2008) Estimation of nucleotide diversity, disequilibrium coefficients, and mutation rates from high-coverage genome-sequencing projects. Molecular Biology and Evolution, 25, 2409-2419.

Mastretta-Yanes A, Arrigo N, Alvarez N et al. (2015) Restriction site-associated DNA sequencing, genotyping error estimation and de novo assembly optimization for population genetic inference. Molecular ecology resources, 15, 28-41.

NOAA (2015, October 15). Corals. Retrieved March 11, 2017, from http://www.nmfs.noaa.gov/pr/species/invertebrates/corals.htm

Noble D (2015) Evolution beyond neo-Darwinism: a new conceptual framework. The Journal of Experimental Biology, 218, 7-13.

Pante E, Abdelkrim J, Viricel A et al. (2015) Use of RAD sequencing for delimiting species. Heredity, 114, 450-459.

Paradis E, Claude J, Strimmer K (2004) APE: Analyses of Phylogenetics and Evolution in R language. Bioinformatics, 20, 289-290.

Patterson C, Williams DM, Humphries CJ (1993) Congruence between molecular and morphological phylogenies. Annual review of ecology and systematics, 24, 153-188.

Paz-García DA, Hellberg ME, García-de-León FJ, Balart EF (2015) Switch between Morphospecies of Pocillopora Corals. The American Naturalist, 186, 434-440.

Peterson BK, Weber JN, Kay EH, Fisher HS, Hoekstra HE (2012) Double digest RADseq: an inexpensive method for de novo SNP discovery and genotyping in model and non-model species. Plos One, 7, e37135. 
548

Potter D, Lajeunesse T, Saunders G, et al. (1997) Convergent evolution masks extensive biodiversity among marine coccoid picoplankton. Biodiversity and conservation, 6, 99-107.

Prada C, DeBiasse MB, Neigel JE et al. (2014) Genetic species delineation among branching Caribbean Porites corals. Coral reefs (Online), 33, 1019-1030.

Putnam HM, Davidson JM, Gates RD (2016) Ocean acidification influences host DNA methylation and phenotypic plasticity in environmentally susceptible corals. Evolutionary applications, 9, 11651178.

Recknagel H, Jacobs A, Herzyk P, Elmer KR (2015) Double-digest RAD sequencing using lon Proton semiconductor platform (ddRADseq-ion) with nonmodel organisms. Molecular ecology resources, 15, 1316-1329.

Robinson MD, McCarthy DJ, Smyth GK (2010) edgeR: a Bioconductor package for differential expression analysis of digital gene expression data. Bioinformatics, 26, 139-140.

Rosser NL, Thomas L, Stankowski S et al. (2017) Phylogenomics provides new insight into evolutionary relationships and genealogical discordance in the reef-building coral genus Acropora. Proceedings. Biological Sciences / the Royal Society, 284.

Rützler K, Macintyre I (1982) The habitat distribution and community structure of the barrier reef complex at Carrie Bow Cay, Belize. In: The Atlantic barrier reef ecosystem at Carrie Bow Cay, Belize, I. Structure and communities. , pp. 9-45.

Santos S, Taylor D, Kinzie R et al. (2002) Molecular phylogeny of symbiotic dinoflagellates inferred from partial chloroplast large subunit (23S)-rDNA sequences. Molecular Phylogenetics and Evolution, 23, 97-111.

Sarda S, Zeng J, Hunt BG, Yi SV (2012) The evolution of invertebrate gene body methylation. Molecular Biology and Evolution, 29, 1907-1916.

Schield DR, Walsh MR, Card DC et al. (2016) EpiRADseq: scalable analysis of genomewide patterns of methylation using next-generation sequencing. Methods in ecology and evolution / British Ecological Society, 7, 60-69.

Schlöder C, Guzman H (2008) Reproductive patterns of the Caribbean coral Porites furcata (Anthozoa, Scleractinia, Poritidae) in Panama. Bulletin of marine science, 82, 107-117.

Schmidt-Roach S, Lundgren P, Miller KJ et al. (2013) Assessing hidden species diversity in the coral Pocillopora damicornis from Eastern Australia. Coral reefs (Online), 32, 161-172.

Sebens KP (1987) The ecology of indeterminate growth in animals. Annual review of ecology and systematics, 18, 371-407.

Shoguchi E, Shinzato C, Kawashima T et al. (2013) Draft assembly of the Symbiodinium minutum nuclear genome reveals dinoflagellate gene structure. Current Biology, 23, 1399-1408.

Skinner MK (2015) Environmental Epigenetics and a Unified Theory of the Molecular Aspects of Evolution: A Neo-Lamarckian Concept that Facilitates Neo-Darwinian Evolution. Genome Biology and Evolution, 7, 1296-1302.

Skinner MK, Guerrero-Bosagna C, Haque MM (2015) Environmentally induced epigenetic transgenerational inheritance of sperm epimutations promote genetic mutations. Epigenetics, 10, 762-771.

Skinner MK, Gurerrero-Bosagna C, Haque MM et al. (2014) Epigenetics and the evolution of Darwin's Finches. Genome Biology and Evolution, 6, 1972-1989.

Smith TA, Martin MD, Nguyen M, Mendelson TC (2016) Epigenetic divergence as a potential first step in darter speciation. Molecular Ecology, 25, 1883-1894.

Smith G, Smith C, Kenny JG, Chaudhuri RR, Ritchie MG (2015) Genome-wide DNA methylation patterns in wild samples of two morphotypes of threespine stickleback (Gasterosteus aculeatus). Molecular Biology and Evolution, 32, 888-895.

Stoddart J (1983) Asexual production of planulae in the coral Pocillopora damicornis. Marine Biology. 
596 Todd PA (2008) Morphological plasticity in scleractinian corals. Biological Reviews of the Cambridge

$597 \quad$ Philosophical Society, 83, 315-337.

598 Weil E (1992) Genetic and morphological variation in Caribbean and eastern Pacific Porites (Anthozoa,

$599 \quad$ Scleractinia). Preliminary results. Proc 7th int coral Reef Symp.

600 Yang Z, Rannala B (2012) Molecular phylogenetics: principles and practice. Nature Reviews. Genetics, 13,

601 303-314.

602 Zemach A, McDaniel IE, Silva P, Zilberman D (2010) Genome-wide evolutionary analysis of eukaryotic

603 DNA methylation. Science, 328, 916-919. 
Table 1. Porites spp. specimen data. For descriptions of habitat types on the Belize Barrier Reef refer to Rützler \& Macintyre (1982).

\begin{tabular}{|c|c|c|c|c|c|}
\hline Sample \# & Coordinates & Habitat & Depth (m) & Symbiont clade & Branch diameter (mm) \\
\hline 101 & N $16^{\circ} 48.126^{\prime}$, W $88^{\circ} 4.729^{\prime}$ & Lower spur \& groove & 7.9 & c & 19.5 \\
\hline 103 & N $16^{\circ} 48.294^{\prime}$, W $88^{\circ} 4.666^{\prime}$ & Inner reef slope & 16.2 & C & 10.5 \\
\hline 104 & N $16^{\circ} 48.294^{\prime}$, W $88^{\circ} 4.666^{\prime}$ & Inner reef slope & 16.5 & C & 9 \\
\hline 105 & N $16^{\circ} 46.792^{\prime}$, W $88^{\circ} 4.599^{\prime}$ & Lower spur \& groove & 9.8 & C & 11.5 \\
\hline 106 & N $16^{\circ} 46.792^{\prime}$, W $88^{\circ} 4.599^{\prime}$ & Lower spur \& groove & 7.0 & C & 17 \\
\hline 107 & N $16^{\circ} 46.821^{\prime}$, W $88^{\circ} 4.521^{\prime}$ & Inner reef slope & 12.5 & C & 11.5 \\
\hline 108 & N $16^{\circ} 48.120^{\prime}$, W $88^{\circ} 4.751^{\prime}$ & Upper spur and groove & 7.0 & NA & 10.5 \\
\hline 109 & N $16^{\circ} 46.821^{\prime}$, W $88^{\circ} 4.521^{\prime}$ & Inner reef slope & 14.6 & C & 10 \\
\hline 110 & N $16^{\circ} 48.146^{\prime}$, W $88^{\circ} 4.658^{\prime}$ & Inner reef slope & 14.0 & C & 14 \\
\hline 111 & N $16^{\circ} 48.120^{\prime}$, W $88^{\circ} 4.751^{\prime}$ & Upper spur and groove & 7.9 & C & 14 \\
\hline 112 & N $16^{\circ} 46.571^{\prime}$, W $88^{\circ} 4.524^{\prime}$ & Lower spur \& groove & 8.5 & C & 13 \\
\hline 114 & N $16^{\circ} 46.798^{\prime}$, W $88^{\circ} 4.511^{\prime}$ & Outer ridge & 15.5 & C & 9 \\
\hline 115 & N $16^{\circ} 48.354^{\prime}$, W $88^{\circ} 4.998^{\prime}$ & Backreef & 1.2 & C & 20 \\
\hline 116 & N $16^{\circ} 48.354^{\prime}$, W $88^{\circ} 4.998^{\prime}$ & Backreef & 1.2 & NA & 10 \\
\hline 117 & N $16^{\circ} 48.354^{\prime}$, W $88^{\circ} 4.998^{\prime}$ & Backreef & 1.2 & A & 13 \\
\hline 118 & N $16^{\circ} 49.645^{\prime}$, W $88^{\circ} 6.364^{\prime}$ & Mangrove & 0.6 & C & 7 \\
\hline 121 & N $16^{\circ} 49.645^{\prime}$, W $88^{\circ} 6.364^{\prime}$ & Mangrove & 0.6 & C & 7.5 \\
\hline 122 & N $16^{\circ} 49.645^{\prime}$, W $88^{\circ} 6.364^{\prime}$ & Mangrove & 0.6 & C & 6 \\
\hline 123 & N $16^{\circ} 48.181^{\prime}$, W $88^{\circ} 4.885^{\prime}$ & Backreef & 0.8 & C & 21 \\
\hline 124 & N $16^{\circ} 48.115^{\prime}$, W $88^{\circ} 4.983^{\prime}$ & Lagoon/cut & 4.6 & C & 16.5 \\
\hline 125 & N $16^{\circ} 46.798^{\prime}$, W $88^{\circ} 4.511^{\prime}$ & Outer ridge & 16.5 & C & 10 \\
\hline 126 & N $16^{\circ} 46.798^{\prime}$, W $88^{\circ} 4.511^{\prime}$ & Outer ridge & 17.4 & C & 26 \\
\hline 127 & N $16^{\circ} 48.115^{\prime}$, W $88^{\circ} 4.983^{\prime}$ & Lagoon/cut & 2.7 & A & 15 \\
\hline 128 & N $16^{\circ} 48.181^{\prime}$, W $88^{\circ} 4.885^{\prime}$ & Backreef & 0.6 & NA & 10 \\
\hline 129 & N $16^{\circ} 48.115^{\prime}$, W $88^{\circ} 4.983^{\prime}$ & Turtlegrass & 0.9 & A & 10.5 \\
\hline 130 & N $16^{\circ} 48.181^{\prime}$, W $88^{\circ} 4.885^{\prime}$ & Backreef & 0.8 & C & 17.5 \\
\hline 131 & N $16^{\circ} 48.181^{\prime}$, W $88^{\circ} 4.885^{\prime}$ & Turtlegrass & 0.5 & A & 10 \\
\hline
\end{tabular}



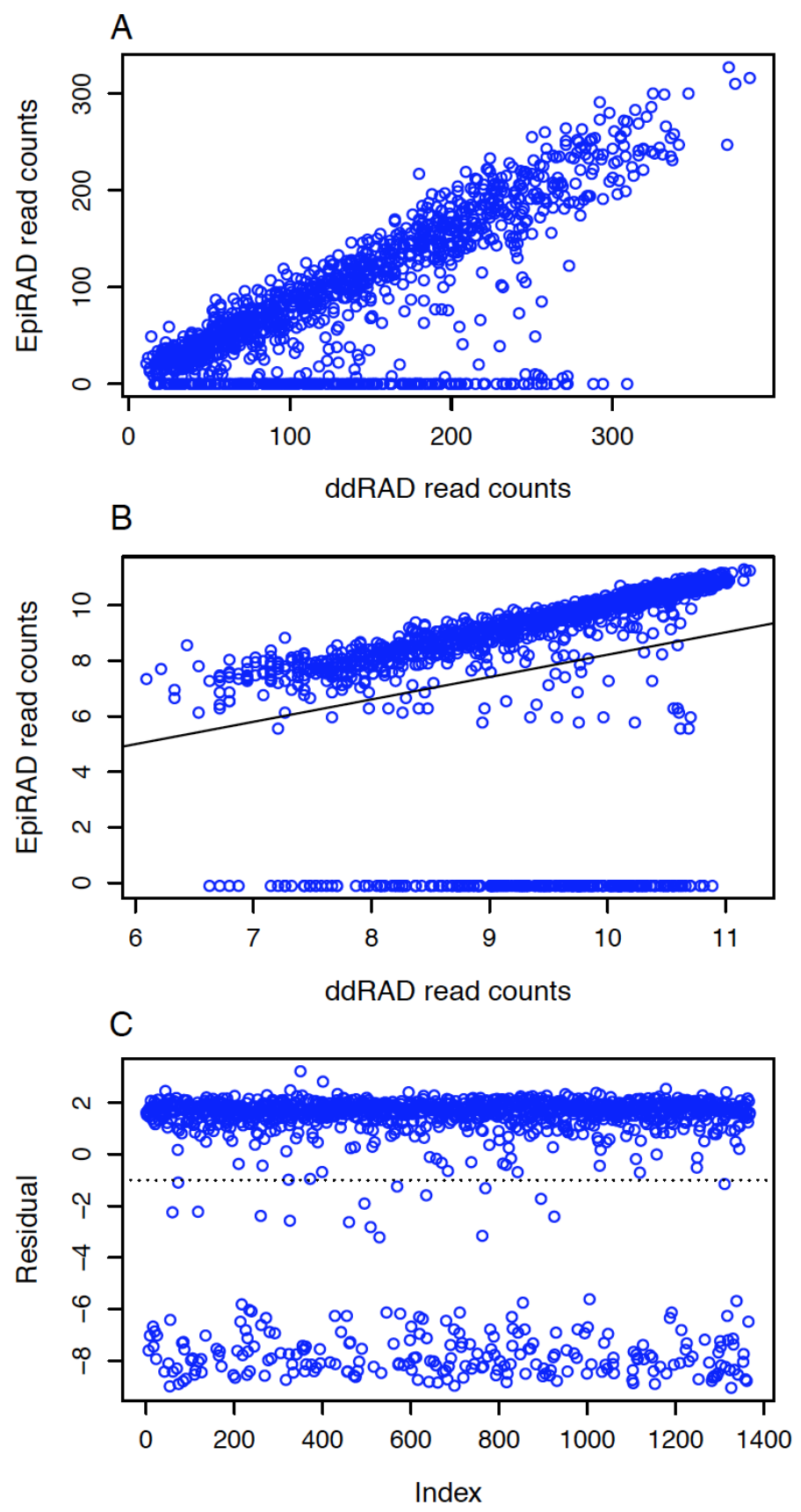

Fig. 1. Determining methylated loci in a representative sample. (A) ddRADseq and EpiRADseq raw read counts for each locus (each point is a locus) were highly correlated except for methylated reads, which were absent or at low abundance in the EpiRAD library and cluster at the bottom of the $y$-axis. (B) ddRADseq and EpiRADseq read counts after TMM normalization, showing regression line from linear model used for derivation of residuals. (C) Residuals from the linear regression of the normalized data. Non-methylated reads have positive residuals and cluster at the top while methylated reads have negative residuals and cluster at the bottom. The binary dataset derived from these data was based on a residual threshold of ? -1 (dotted line) for designating loci as methylated. 

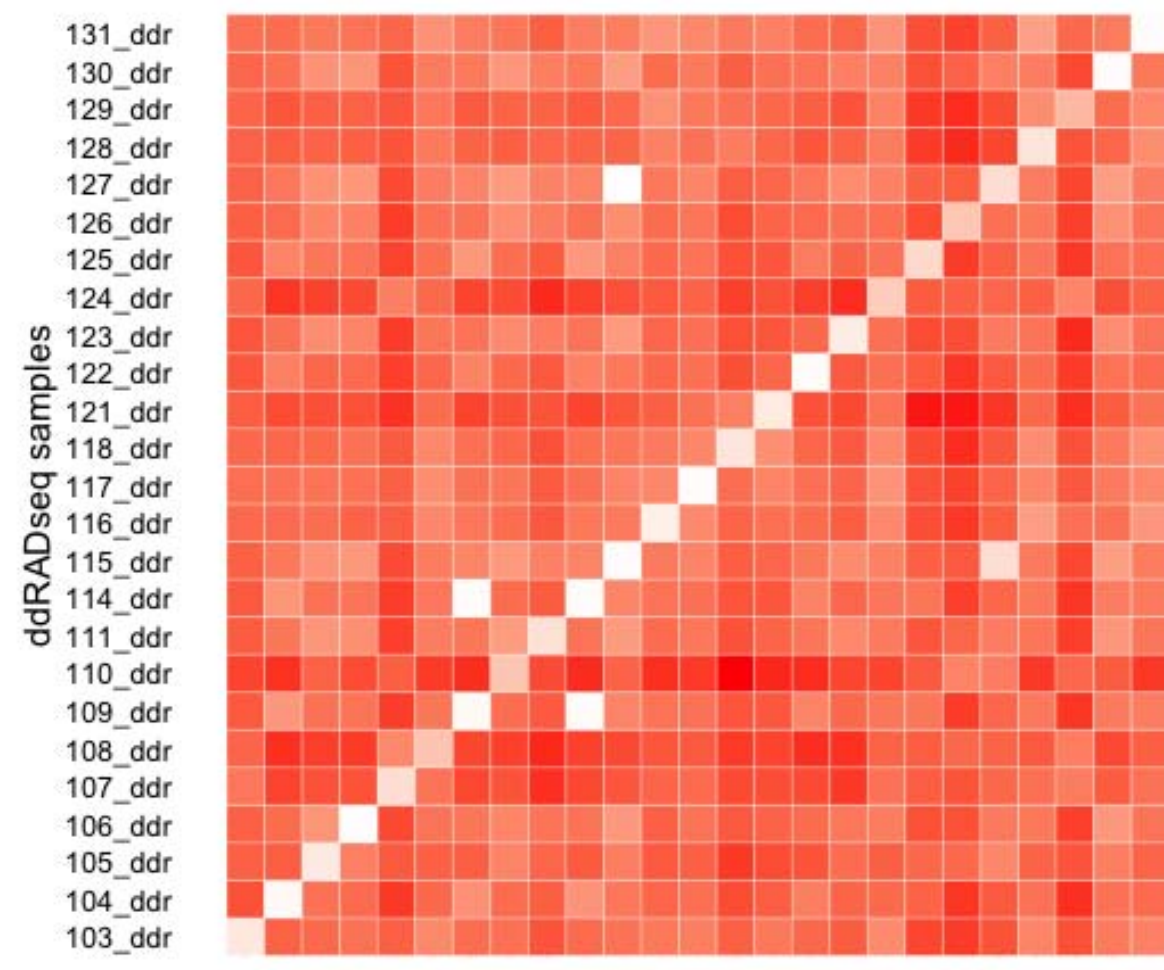

\section{SNP Mismatches}

0.3

0.2

0.1

0.0

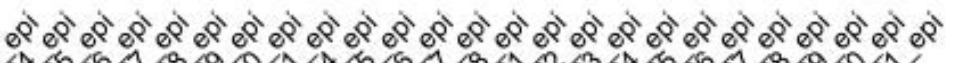

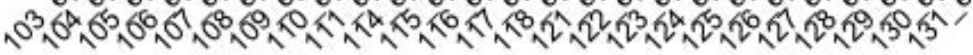

EpiRADseq samples

Fig. 2. Pairwise comparisons of SNP mismatches between all 25 individuals with both ddRADseq and EpiRADseq data. The color ramp represents the proportion of SNP mismatches, also known as genetic distance, between pairs. ddRADseq and EpiRADseq data for the same individual were used as technical replicates to estimate the SNP error rate and are expressed along the diagonal. Two pairs of outliers with a low proportion of mismatches $(109 / 104,115 / 127)$ are possible clones. 
bioRxiv preprint doi: https://doi.org/10.1101/119156; this version posted March 22, 2017. The copyright holder for this preprint (which was not certified by peer review) is the author/funder, who has granted bioRxiv a license to display the preprint in perpetuity. It is made available under aCC-BY-NC-ND 4.0 International license.

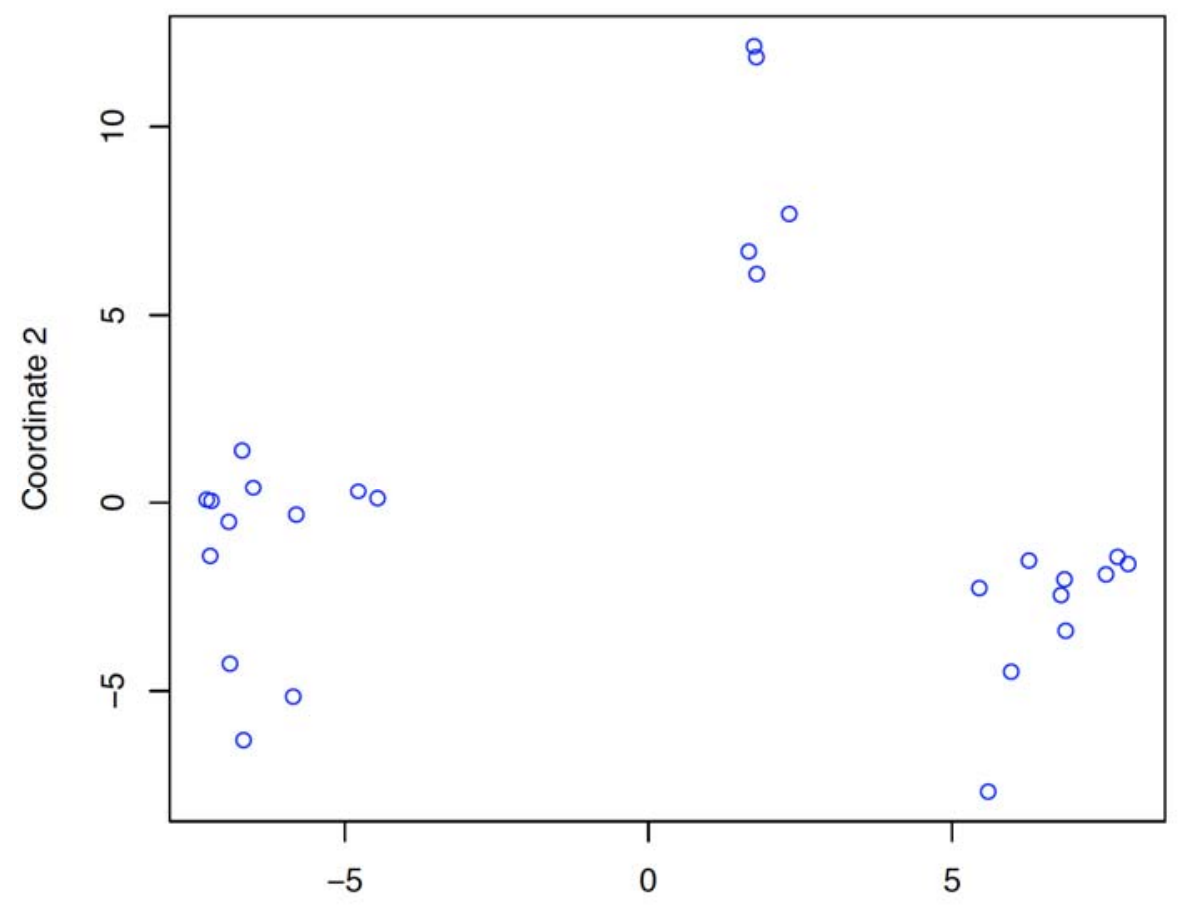

Coordinate 1

Fig 3. Multidimensional scaling plot of multilocus genotypes derived from SNPs in the Porites spp. specimens. Each point represents a specimen. Distances between points represent Euclidean distances projected in two dimensions. 


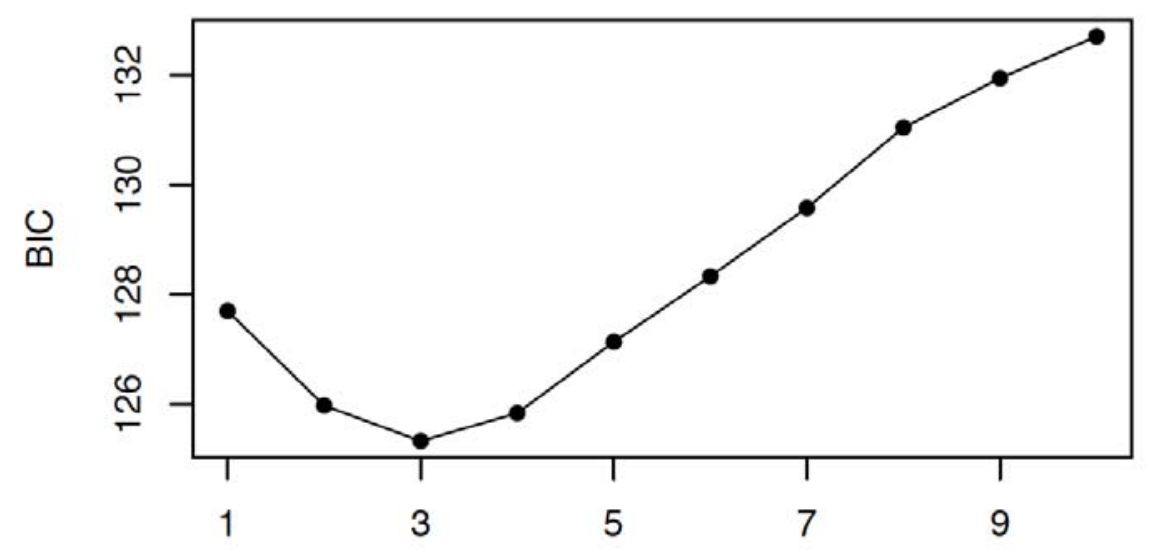

Groups (K)

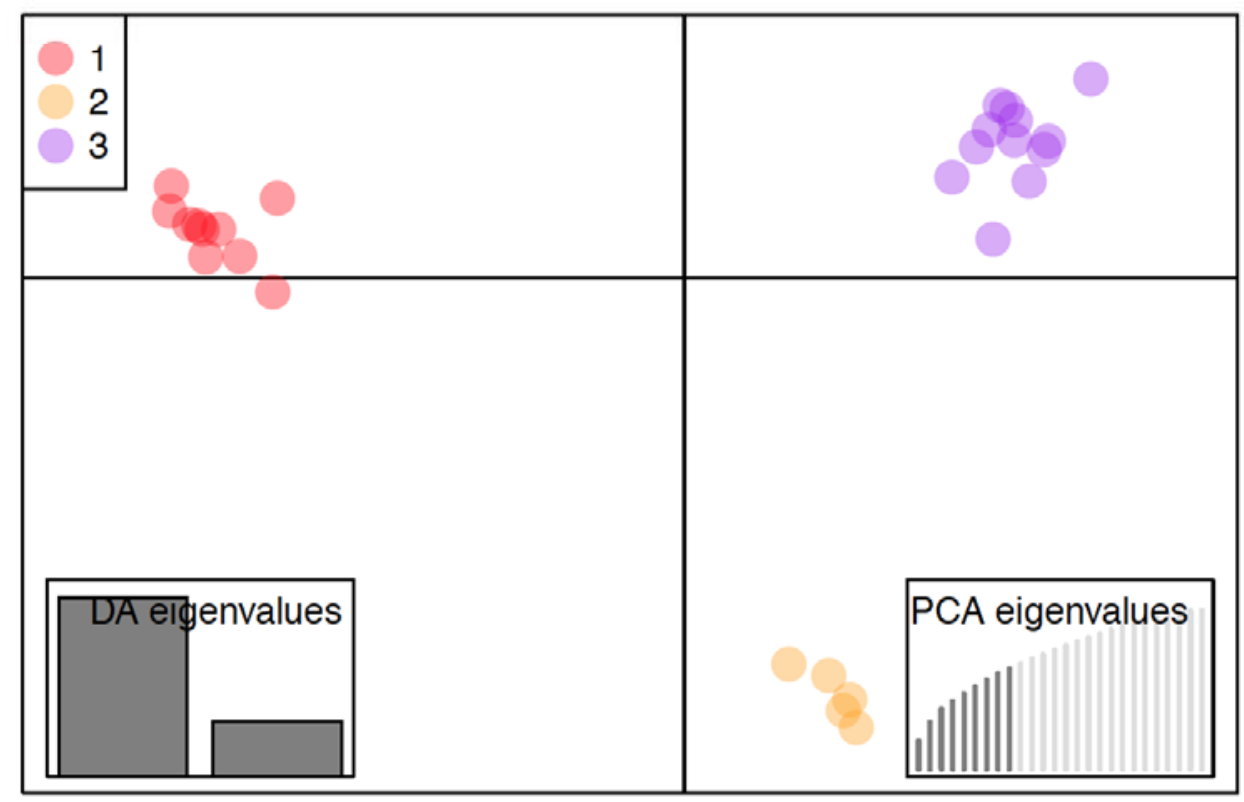

Fig. 4. DAPC analysis of SNP data. (Top) Results of find.clusters analysis on SNP data identifying optimal number of groups. $\mathrm{K}=3$ groups was determined to be optimal based on the lowest value of the Bayesian information criterion (BIC). (Bottom) Results of DAPC analysis on SNP data using the three optimal groups defined by find.clusters. Left inset shows DA eigenvalues illustrating the relative weight of the two DA axes ( $x=D A 1, y=D A 2)$. Right inset shows the relative amount of the variance explained by the 9 principal components used in the DAPC. 


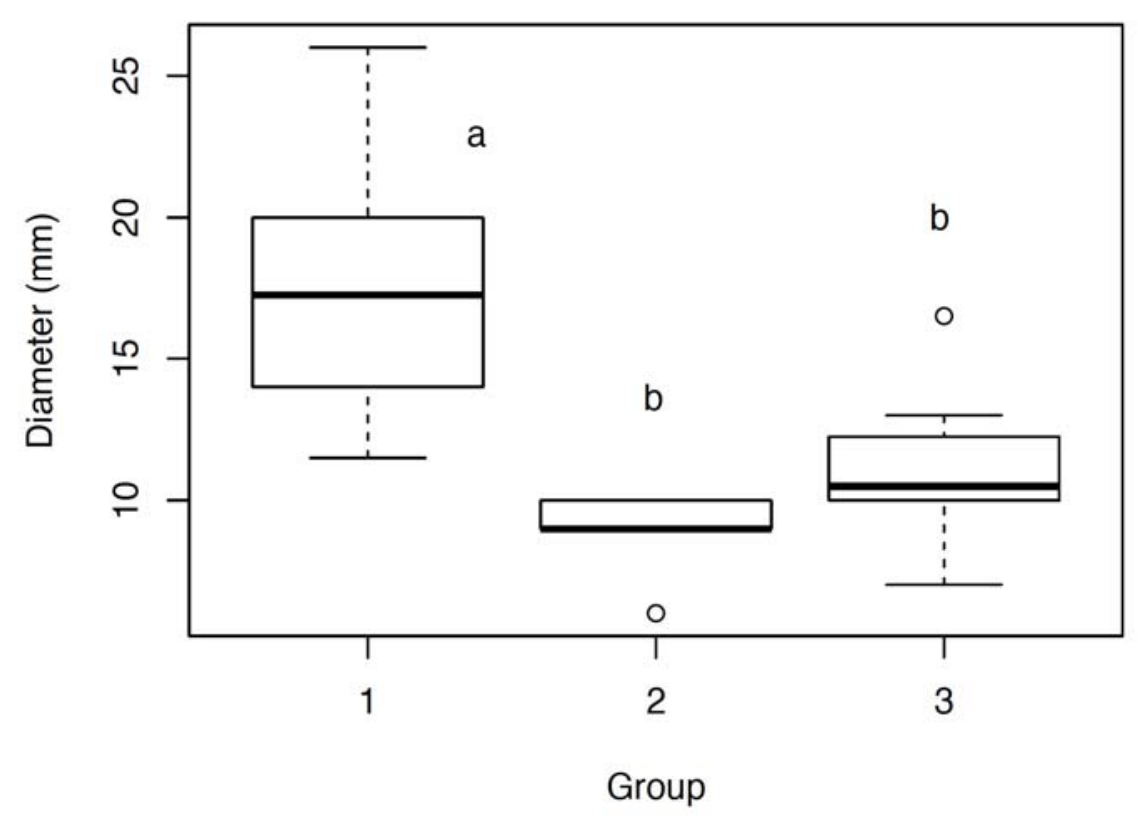

Fig. 5. Comparison of branch diameter in the three Porites groups identified by the DAPC analysis in Fig. 4. Boxes show the median (black horizontal bars) plus the 75th percentile and minus the 25th percentile; whiskers show these percentiles plus or minus 1.5 times the interquartile range. Extreme values are shown as points beyond these ranges. Different letters denote groups determined to be significantly different in pairwise t-tests. 


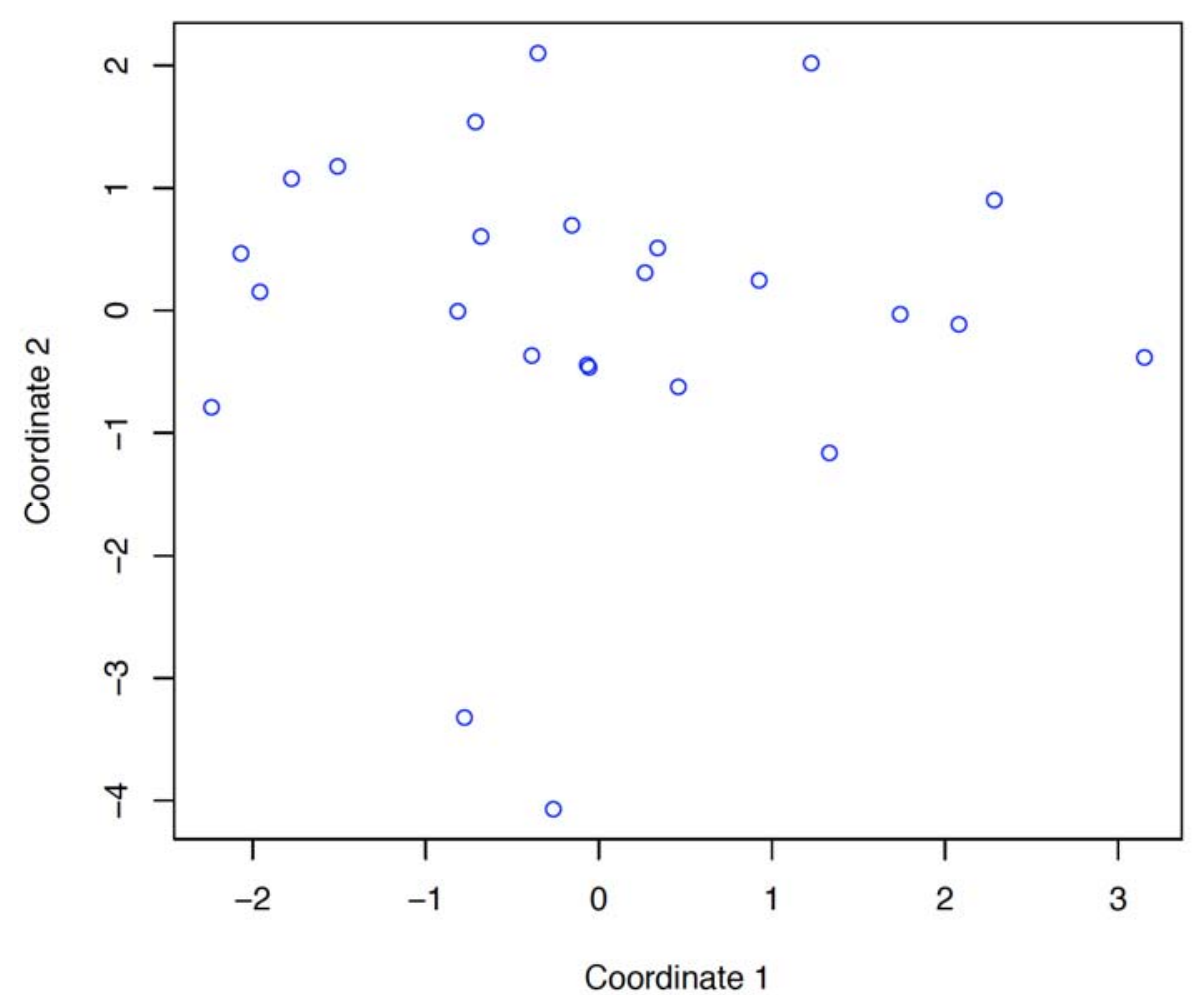

Fig. 6. Multidimensional scaling plot of multilocus epi-genotypes in the Porites spp. specimens. Each point represents a specimen. Distances between points represent Euclidean distances projected in two dimensions. 


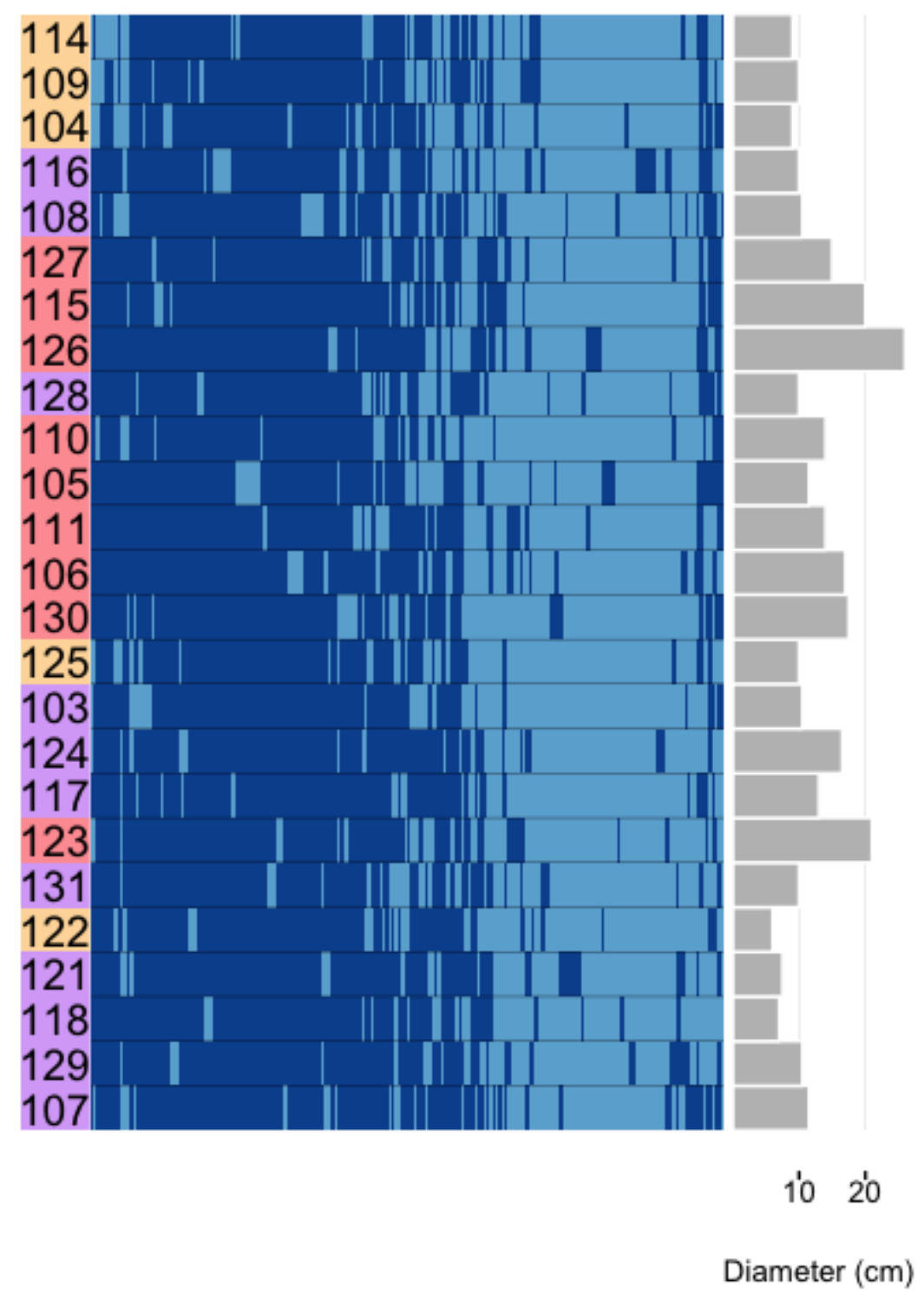

Fig. 7. Heatmap of differentially methylated CpGs. Methylated CpGs are shown in dark blue. Hierarchical clustering was used for ordering of both rows (samples) and columns (CpGs). On the left hand side of the plot, samples are colored by the three SNP groups from the DAPC in Fig. 4. On the right side, a bar plot of the branch diameter of each sample is shown. 


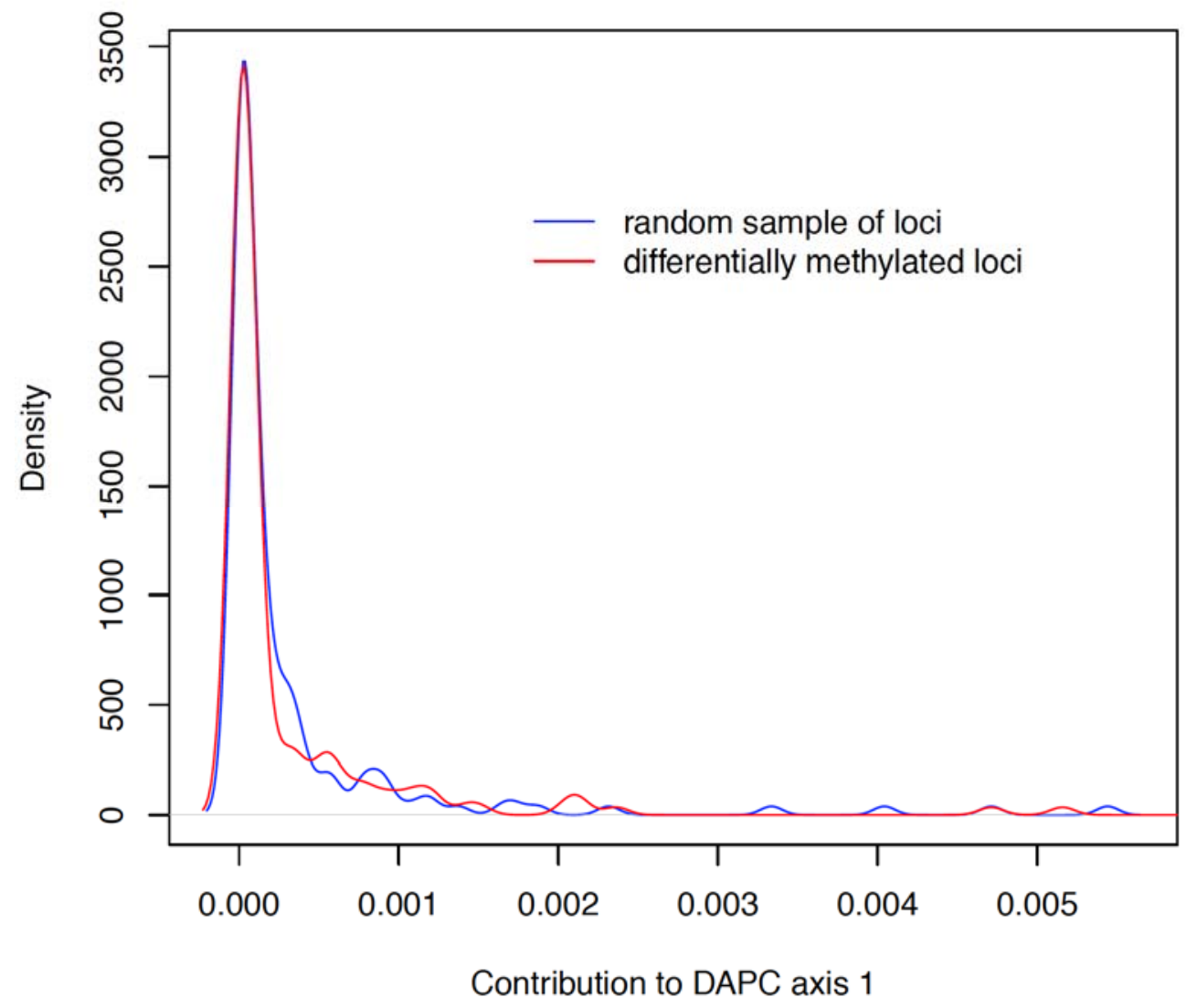

Fig. 8. Distribution of SNP contribution scores to discriminant axis one of the DAPC model in Fig. 3. The red line shows scores of SNPs linked to differentially methylated cut sites on the same loci, while the blue line shows scores from a random sample of the data. 


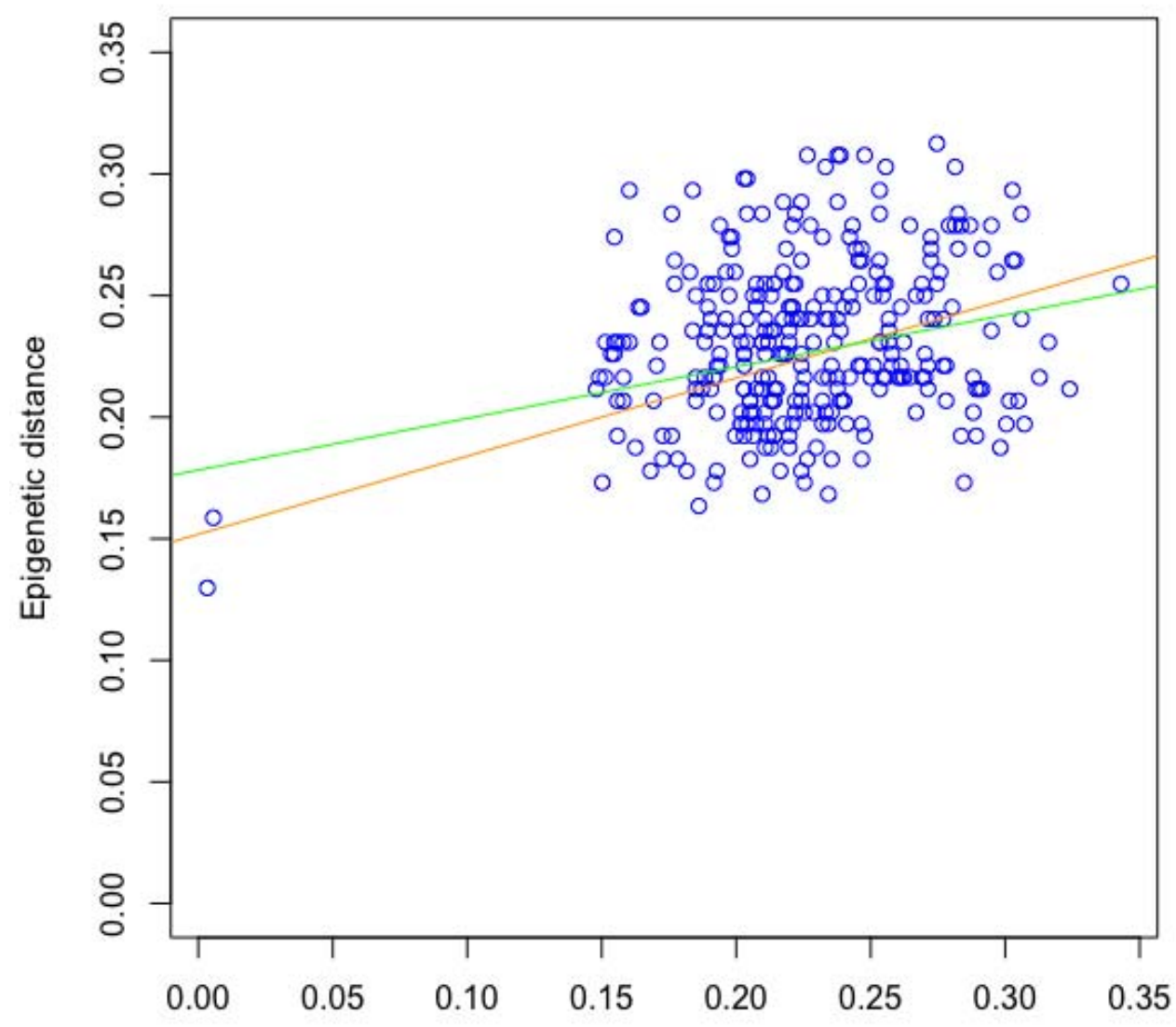

Genetic distance

Fig. 9. Association between pairwise genetic distance and pairwise epigenetic distance. Each point represents a unique pairwise comparison between two samples among the 25 samples that had both genetic and epigenetic data (300 unique pairwise comparisons total). For a given pair, genetic and epigenetic distances represent the proportion of mismatches between multilocus genotypes (SNPS) and multilocus epi-genotypes (CPG methylation status), respectively. The two pairs of outliers are samples 109/114 and 127/115; these possible clones showed the lowest levels of both genetic and epigenetic differentiation. Linear regressions of all the data (orange line, $p<0.001$ ) and exclusive of the outliers (green line, $p=0.002$ ) are shown. 